\title{
NUMERICAL STUDY OF HEAT TRANSFER ENHANCEMENT IN LAMINAR AND TURBULENT FLOW IN ENHANCED TUBES WITH UNIFORM AND NON-UNIFORM THERMAL BOUNDARY CONDITIONS
}

\author{
Matthew Sandlin,, ${ }^{1, *}$ Kashif Nawaz, ${ }^{1}$ Anthony Jacobi, ${ }^{2}$ Xiaofei Wang ${ }^{2}$ \\ ${ }^{1}$ Oak Ridge National Laboratory, Oak Ridge, Tennessee, 37830, United States of America \\ ${ }^{2}$ University of Illinois at Urbana-Champaign, Champaign, Illinois, 61820, United States of America
}

\begin{abstract}
Various types of tube enhancements have been proposed in order to increase heat transfer while minimizing any associated increases in pressure drop. This paper presents a numerical study of two different types of enhanced tubes: the relatively common twisted tape insert tube, and a novel tube with airfoil shaped pins on the inner surface. Each tube is studied in both the laminar and turbulent flow regime. In addition to the typical constant temperature and constant flux boundary conditions, a non-constant heat flux boundary condition is applied on one half of the tube, a situation which may be encountered when absorbing solar radiation. In general, each type of enhanced tube offers a thermal performance factor greater than unity at certain Re. In addition, the non-uniform heat flux boundary condition appears to function similarly as the uniform heat flux boundary condition.
\end{abstract}

KEY WORDS: numerical heat transfer, computational fluid dynamics, heat transfer enhancement

\section{INTRODUCTION}

Heat exchangers are very common in many engineering processes, including residential heating and cooling, chemical processing, waste heat recovery, and power generation. There are often many conflicting constraints that factor into a specific heat exchanger design, such as size, cost, operating temperatures, and material compatibility. Due to increased awareness of resource utilization and the effects of man-made pollution, the ability to improve heat exchanger thermal performance without a large increase in pumping losses is a very active area of research. One area of specific study is to enhance flow mixing inside tubes and channels, increasing the coefficient of convective heat transfer $h$, and thus, the total amount of heat transferred between fluid streams $[8,12,18]$.

Two of the methods of enhancing heat transfer inside tubes are to use inserts, and to modify the surface of the tube itself. One popular form of insert is a so-called twisted tape insert. Twisted tape inserts can take many

This manuscript has been authored in part by UT-Battelle, LLC, under contract DE-AC05-00OR22725 with the US Department of Energy (DOE). The US government retains and the publisher, by accepting the article for publication, acknowledges that the US government retains a nonexclusive, paid-up, irrevocable, worldwide license to publish or reproduce the published form of this manuscript, or allow others to do so, for US government purposes. DOE will provide public access to these results of federally sponsored research in accordance with the DOE Public Access Plan (http://energy.gov/downloads/doe-public-access-plan). 
forms, including alternating twisted tapes [1], perforated twisted tapes [5], twisted tapes with various cuts and louvers [14], twisted tapes that don't span the entire inner flow channel [9], and twisted tapes with a tapered axial dimension $[16,17]$. However, the simplest case is a twisted tape that spans the entire inner diameter of the tube [11]. In general, twisted tape inserts increase convective heat transfer by introducing swirl into the flow in a tube, which increases turbulence and flow mixing [8].

As with twisted tape inserts, there are many different methods of altering the tube surface to enhance heat transfer, such as with dimples [13], spiral grooves [4], and corrugations [2]. Flat channels are also good candidates for surface modification, as a very wide array of surface modifications can be made relatively easily [6]. One geometry of particular interest that has been studied for flat plate heat exchangers is an airfoil pin, which is typically based on a NACA profile [7]. These pins are typically made with either an optical lithography technique (as in printed circuit heat exchangers) or using additive manufacturing methods [10]. The use of additive manufacturing makes it feasible to study the effects of airfoil pins on the inner surface of round tubes.

This study has two main goals. The first goal to numerically investigate the performance of several types of enhanced tubes in both the laminar and turbulent regime to identify if certain designs are better suited for either forced laminar or turbulent heat transfer. The second goal is to investigate the effects of three different thermal boundary conditions, which are the typical constant wall temperature and heat flux boundary conditions, and a non-uniform thermal flux boundary condition that could be caused by incident (concentrated) solar radiation.

\section{PHYSICAL MODEL}

This section will describe the physical domain and boundary conditions used in the numerical simulations, as well as the parameters of interest.

\subsection{Twisted tape insert}

The twisted tape insert was modeled as a tube with an inner diameter of $4.23 \mathrm{~mm}$. The twist pitch of the tape was kept at $8.46 \mathrm{~mm}$, or twice the inner diameter. Three different design variants were used: a twisted tape that covers the entire tube diameter, a twisted tape with a $2.12 \mathrm{~mm}$ gap down the center (referred to as "outer"), and a twisted tape consisting only of the $2.12 \mathrm{~mm}$ center tape (referred to as "inner"). The tape was 0.1224 $\mathrm{mm}$ thick. The total length of the simulated tube was $16.92 \mathrm{~mm}$, or one complete rotation of the tape. The tube thickness was set at $1 \mathrm{~mm}$ (although this was mostly for giving a uniform outer surface for calculating boundary conditions; see subsection 2.3). Except for the "inner" design, the twisted tapes are assumed to be part of the tube wall, with no thermal contact resistance.

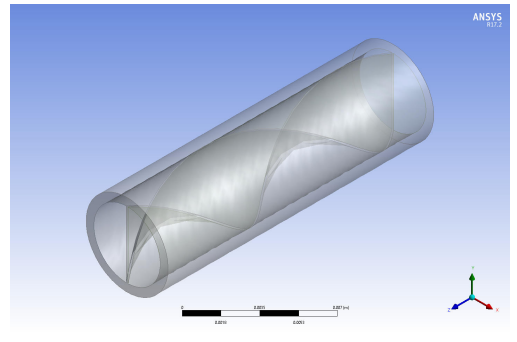

(a) Twisted tape

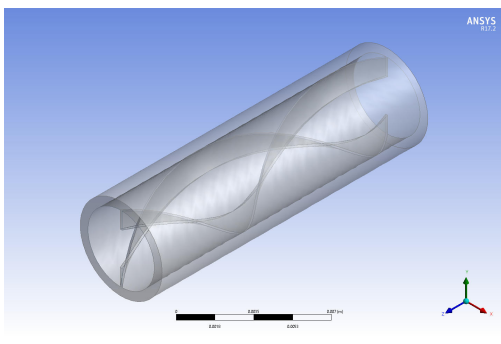

(b) Twisted tape "outer"

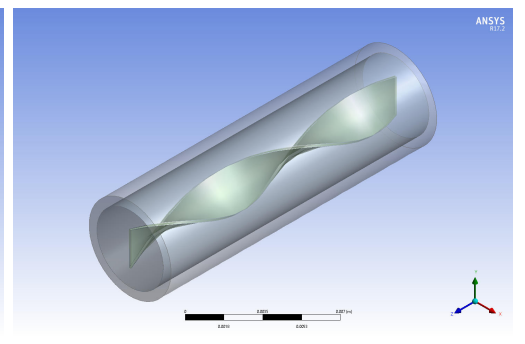

(c) Twisted tape "inner"

Fig. 1 Different twisted tape configurations.

\subsection{Airfoil in tube}

The airfoil-in-tube concept was modeled as an elliptical tube with $9 \mathrm{~mm}$ and $3 \mathrm{~mm}$ major and minor diameter respectively. The inner surface of the tube is covered in alternating rows of small airfoil shaped pins. The 
airfoils are NACA0020 profiles, with chord lengths of $1 \mathrm{~mm}$, and a $0.25 \mathrm{~mm}$ height. There are 22 fins around the inner diameter of the tube, and each row of pins is separated by $1 \mathrm{~mm}$ from trailing to leading edge. Each row is staggered so that every pin is in the center of the gap between the immediate two upstream pins. The total length of the simulated tube was $4 \mathrm{~mm}$, or two complete rows of pins. An smooth elliptical tube was also modeled for comparison purposes.

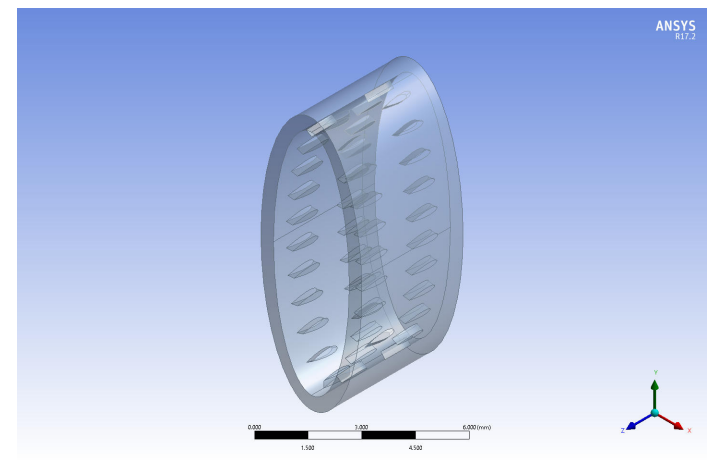

Fig. 2 Elliptical tube with airfoil pins.

\subsection{Boundary conditions}

All tube designs had identical boundary conditions. The tubes were modeled with periodic boundary conditions on the inlet and outlet of the tube, allowing the use of a smaller numerical domain while avoiding entrance length issues. The bulk fluid upstream temperature was set at $300 \mathrm{~K}$. All fluid/surface boundaries were modeled as no slip boundaries, and were treated as thermally coupled. Three different thermal boundary conditions were given to the outer tube surface: a constant temperature boundary condition, a uniform flux boundary condition, and a non-uniform flux boundary condition which is given by

$$
q= \begin{cases}0 & \text { if } \mathbf{a} \cdot \mathbf{x}<0 \\ q_{o} \frac{\mathbf{x} \cdot \mathbf{a}}{\|\mathbf{a}\| \mid\|\mathbf{x}\|} & \text { otherwise }\end{cases}
$$

where $\mathbf{a}$ and $\mathbf{x}$ are the vectors that represent an incoming direction and the normal of the outer tube surface, and $q_{o}$ is the maximum heat flux. In this way, this boundary condition simulates an incoming solar radiation heat flux (and is referred to as "solar" throughout this paper); the non-heated side of the tube is treated as insulated. The constant wall boundary was set at $350 \mathrm{~K}$ for the twisted tape tubes, and at $310 \mathrm{~K}$ for the airfoil pin tube as the periodic solver was unable to handle such a large temperature difference along a shorter simulated tube. The corresponding heat flux boundary conditions were calculated from the average heat flux through the outer tube surface of the constant temperature case in order to maintain a similar solution scale across boundary conditions. Figure 3 shows how the solar boundary condition was applied to both axes of an elliptical tube.

\subsection{Parameters}

The thermal hydraulic performance of each tube design is calculated at a given Reynolds number Re, given by

$$
R e=\frac{\rho u D_{h}}{\mu}
$$

where $\rho$ is the fluid density, $u$ is the velocity, $D_{h}$ is the hydraulic diameter given by four times the flow area 

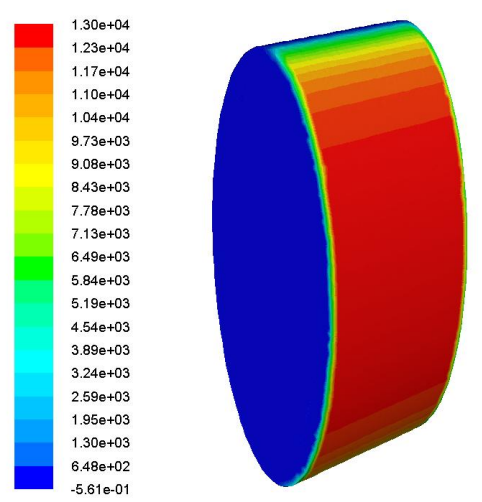

(a) Aligned with the major axis
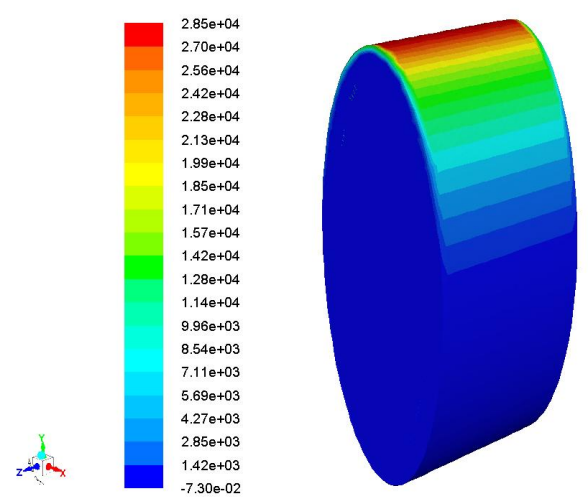

(b) Aligned with the minor axis

Fig. 3 How the non-uniform flux boundary condition looks when applied to the elliptical tube. Note the different maximum value on each scale.

divided by the wetted perimeter of a tube, and $\mu$ is the dynamic viscosity. The friction coefficient $f$ of a given tube is calculated by

$$
f=\frac{2 D_{h}}{\rho u^{2}} \frac{\Delta p}{L}
$$

where $\Delta p / L$ is the change in pressure per unit length of tube.

To evaluate the heat transfer performance of a given tube, the Nusselt number $N u$ is calculated by

$$
N u=\frac{h D_{h}}{k}
$$

where $h$ is the coefficient of convective heat transfer, and $k$ is the fluid thermal conductivity. In this study, $h$ is evaluated at the area averaged tube inner surface temperature and heat flux, and the volume average fluid temperature.

To assess the thermal performance of a given tube, the performance evaluation criteria (PEC) is defined as [19]

$$
\eta=\frac{N u / N u_{o}}{\left(f / f_{o}\right)^{1 / 3}}
$$

where the subscript $o$ refers to a smooth circular tube. Thus, any tube design with a PEC $>1$ has a larger increase in heat transfer than the corresponding increase in pumping power required due to increased internal friction and pressure loss (such as through fins).

\section{MATHEMATICAL MODEL}

\subsection{Governing equations}

This study used the finite volume method (FVM), which is a standard technique of computational fluid dynamics (CFD) [15]. It was implemented in Ansys/FLUENT v17, which is a commercial CFD code. For an imcompressible, flow with constant property fluids, FLUENT solves the following conservation equations [3]: 
Mass conservation:

$$
\nabla \cdot \mathbf{u}=0
$$

where $\mathbf{u}$ is the mean velocity.

Momentum conservation:

$$
\nabla \cdot(\rho \mathbf{u u})=-\nabla p+\nabla \cdot \overline{\bar{\tau}}
$$

where $\rho$ is the density, $p$ is the pressure, and $\overline{\bar{\tau}}$ is the stress tensor.

Energy conservation:

$$
\nabla \cdot(\mathbf{u}(\rho E+p))=\nabla \cdot\left(k_{e f f} \nabla T+\overline{\bar{\tau}} \cdot \mathbf{u}\right)
$$

where

$$
\begin{gathered}
E=h-\frac{p}{\rho}+\frac{v^{2}}{2}, \\
h=\int_{T_{r e f}}^{T} c_{p} d T
\end{gathered}
$$

$c_{p}$ is the constant pressure specific heat, $T$ is the temperature, and $k_{e f f}$ is the effective thermal conductivity, which depends on the specific flow model (i.e. laminar or turbulent) used.

For modeling laminar flow, the right hand term in Equation 7 simplifies to $\mu \nabla^{2} \mathbf{u}$, where $\mu$ is the viscosity. For modeling turbulent flow, the term becomes $\nabla\left(\mu\left(\nabla \mathbf{u}-\rho \overline{u^{\prime} u^{\prime}}\right)\right)$, where $\rho \overline{u^{\prime} u^{\prime}}$ represents the Reynolds stresses. To model the turbulence, the ReNormalization Group (RNG) $k-\epsilon$ model was used. The RNG $k-\epsilon$ model is a two equation model that discretizes the turbulent kinetic energy, $k$, and its dissipation rate, $\epsilon$. In this case, the Boussinesq hypothesis is used to relate the Reynolds stresses to the mean velocity gradients, and the equations for $k$ and $\epsilon$ are given as

$$
\begin{gathered}
\nabla(\rho k \mathbf{u})=\nabla\left(\alpha_{k} \mu_{e f f} \nabla k\right)+G_{k}-\rho \epsilon \\
\nabla(\rho \epsilon \mathbf{u})=\nabla\left(\alpha_{\epsilon} \mu_{e f f} \nabla \epsilon\right)+C_{1 \epsilon} \frac{\epsilon}{k} G_{k}-C_{2 \epsilon} \rho \frac{\epsilon^{2}}{k}-R_{\epsilon}
\end{gathered}
$$

where $C_{1 \epsilon}$ and $C_{2 \epsilon}$ are constants, $G_{k}$ represents the production of kinetic energy, and $R_{\epsilon}$ is defined in [20].

\subsection{Numerical Methods}

Each simulation was solved with the finite volume method. The SIMPLE algorithm [15] was used to achieve pressure-velocity coupling. Gradients were computed using a Green-Gauss node based scheme. Pressure was computed using the PRESTO! algorithm, and the momentum, turbulence quantities, and energy equations were solved with second order schemes. Simulations were started without considering heat transfer; these were considered converged when the continuity and momentum residuals had dropped below $10^{-5}$; due to residual stalling and possible oscillatory behavior, an alternative convergence criteria of $10^{-6}$ on the average velocity passing through a plane midway between the two ends of the domain was used.

Once flow convergence was reached, the momentum and turbulence equations were disabled while the energy equation was enabled, allowing for each thermal run to proceed from identical initial conditions. The energy equation was considered converged when the residuals dropped below $10^{-8}$, or when the surface area averaged residual of the wall heat flux at the inner tube surface was below $10^{-6}$. 


\subsection{Meshing and grid independence}

All meshing was done in the Ansys meshing application. Due to the complex geometries considered, unstructured tetrahedral meshes were used. Element size was decreased around sharp geometric boundaries, such as outside corners, and refinement layers were applied to all solid wall boundaries in order to maintain a first node height of approximately $y^{+}=1$. Due to the two different geometries under consideration, grid independence studies were performed on the full twisted tape geometry and the airfoil pin geometry. It was found that both systems became roughly grid independent when the maximum element size was reduced to $10 \%$ of $D_{h}$. The total element counts ranged from approximately 450000 for the twisted tape case, to approximately 2000000 for the airfoil pin case. Some examples of the meshes used for this study are show in Figure 4.

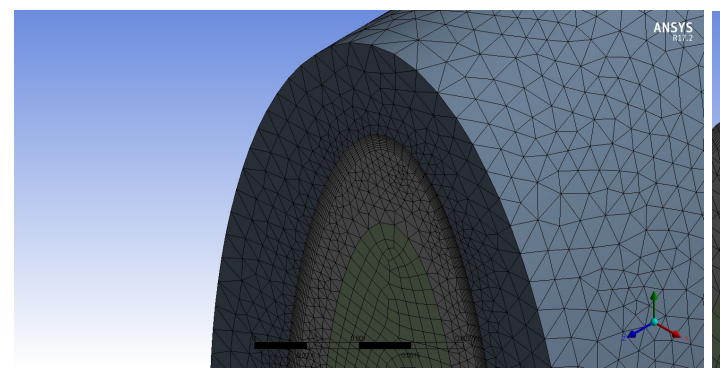

(a) The elliptical airfoil pin fin mesh geometry

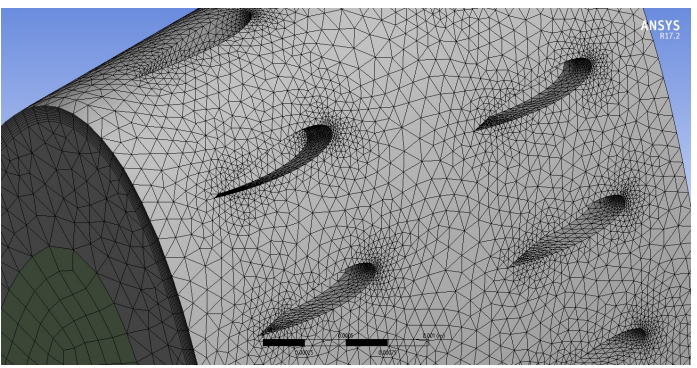

(b) Details of the mesh around the pins

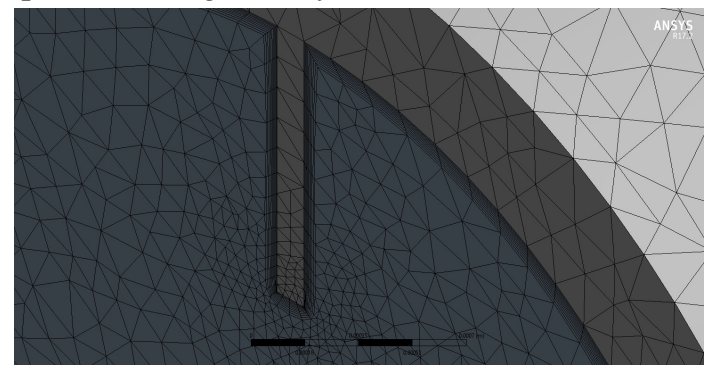

(c) Details of the mesh for the outer twisted tape geometry, showing the boundary layers

Fig. 4 Examples of the tetrahedral meshes used in this study.

\section{RESULTS}

\subsection{Friction factor}

Figure 5 shows the results of how $f$ varies with $R e$ for the geometries considered. The regular and outer twisted tape tubes appear to have a maximum friction factor increase somewhere near the transitional flow regime. The laminar values for the twisted tape geometries are somewhat higher than others have reported in the literature, but the effect of twist ratios on these parameters is significant, and this study uses smaller twist ratios than in the other sources (the choice of turbulence model can have a large impact on predicted values at higher $R e$ as well). In contrast, the friction factor for the airfoil pins appear to be monotonically increasing, but potentially approaching a maximum. This is likely due to the impact a particular design has on interrupting the boundary layer; the twisted tape designs, especially the full coverage and outer designs, reshape the boundary layer entirely, while the airfoil pins merely protrude into it. The inner twisted tape, while generating its own boundary layer and associated drag along its surface, does not disturb the boundary layer forming at the tube wall as much as the other two twisted tape designs. The plain elliptical tube appears to induce more drag than a plain circular tube in laminar flow, but suffers less drag in turbulent flow; however the difference is very small, and could still be within the limits of numerical error. It should be noted that, for $R e$ greater than 1000 , both the regular and twisted tape geometries did not converge on the momentum or turbulence residuals. It 
is suspected that there is some sort of oscillatory flow feature being induced by the relatively tight twist ratio of the designs that is not able to be captured by a steady state solver. In addition, running the two previously mentioned geometries with a turbulent solver and as a transient simulation did not produce markedly different results, nor aide in convergence. More investigation for these two specific geometries may be warranted.

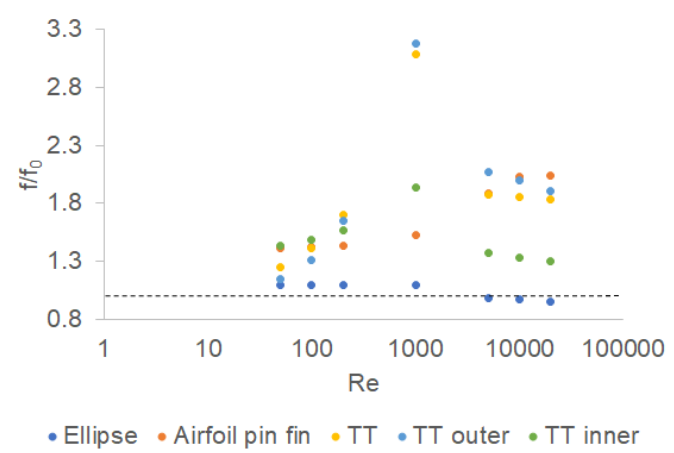

Fig. 5 Ratio of $f$ versus $R e$.

\subsection{Nusselt number}

Figure 6 shows the results of how $N u$ varies with $R e$ for the three thermal boundary conditions. The results compared to a plain tube are similar for each given geometry compared across boundary conditions. Again, the laminar values for the twisted tape geometries are somewhat higher than reported in the literature. When comparing thermal performance, the non-uniform flux boundary condition typically behaves more like the uniform flux boundary condition; it is expected that the performance difference between these boundary conditions will shrink as the thermal conductivity of the tube material goes up. In reality, this will also depend on the tube material's optical properties, as well as the heat loss conditions on the non-irradiated side of the tube. For the elliptical cases, the solar flux applied normal to the major axis performed better than when applied normal to the minor axis, and is what is plotted. This is due to the larger amount of surface area being close to perpendicular to the incoming radiation, and the shorter distance energy needs to diffuse from the heated side of the tube into the fluid stream.

\subsection{Performance enhancement criteria}

Figure 7 shows the PEC of each geometry under the various thermal boundary conditions considered. Clearly, the regular and outer twisted tape designs offer relatively large increases in performance in the higher laminar flow regimes, but suffer too much excess pressure loss in turbulent flow to make up for the increase in heat transfer. In contrast, the airfoil pin fins may offer increased performance at lower turbulent flows; parametric studies need to be conducted to assess the impact of varying pin geometry and placement on heat transfer and pressure drop. The inner twisted tape design only shows a PEC greater than unity in the upper laminar flow regime, although the size of the tape (i.e., the amount of the channel width the tape spans) has a large impact on its performance, and this is a somewhat narrow tape. Overall, this study calculated lower PEC at every $R e$ except 1000 than in other studies, but overall trends are similar to other findings.

\section{CONCLUSIONS AND FUTURE WORK}

Heat transfer and friction factors of various internally enhanced tube designs have been numerically studied in both laminar and turbulent flow. Three thermal boundary conditions were considered: constant temperature, uniform flux, and a non-uniform flux which was proportional to the angle between the tube surface and a given direction. A performance enhancement criteria was calculated for each design variant. In summary: 


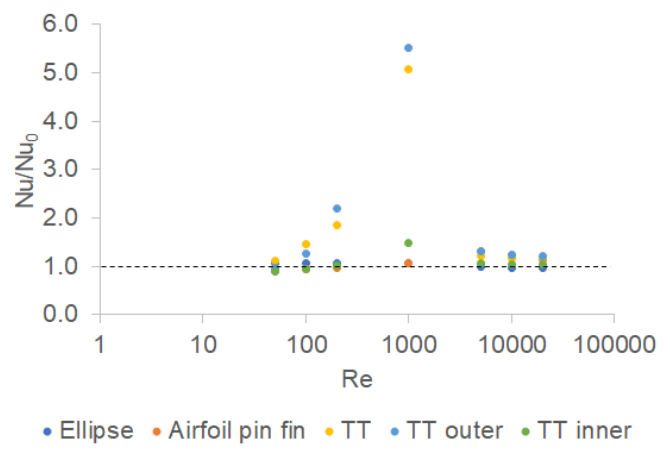

(a) isothermal

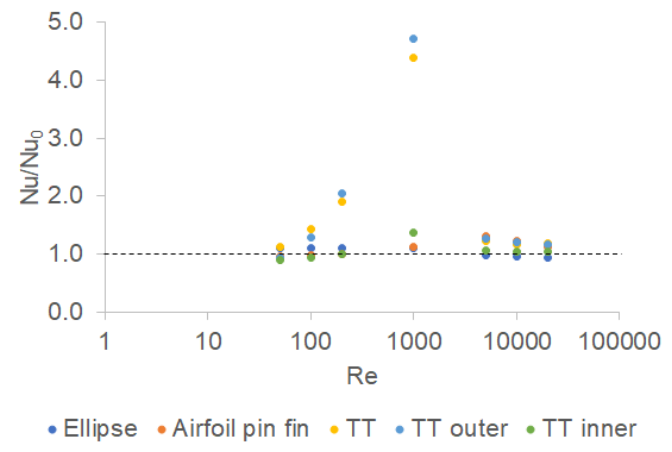

(b) isoflux

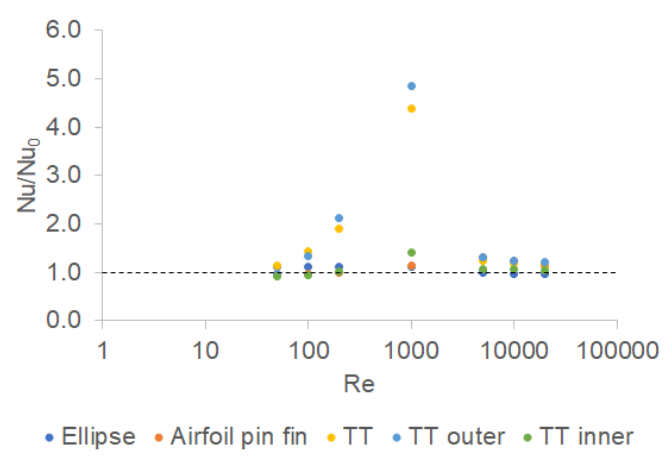

(c) solar

Fig. $6 N u$ versus $R e$ for different thermal boundary conditions.

1. Except for the smooth elliptical tube, all geometries considered had higher friction factors than a smooth circular tube, which is expected. The smooth elliptical tube may have a slightly smaller pressure loss at turbulent flows than a circular tube. All of the twisted tape designs had a peak friction factor increase at an $R e$ of 1000. The elliptical airfoil pin tube had a monotonically increasing friction factor. Possible oscillatory behavior for the regular and outer twisted tape designs made convergence very difficult or unobtainable via the standard momentum (and turbulence) residuals.

2. Except for the inner twisted tape design, all of the modeled tubes offered increased heat transfer performance over a wide range of $R e$ for all thermal boundary conditions. By far, the largest increases in heat transfer were from the regular and outer twisted tape designs, which showed increases of up to $500 \%$ in $N u$ performance at an $R e$ of 1000 . This could be very useful for a heat exchanger designer if overall volume is the main constraint. The smooth elliptical tube appears to only perform better than a smooth circular tube in laminar flow, while the elliptical airfoil pin tube only performs better in turbulent flow. The inner twisted tape tube only offers better performance at an Re of 1000, likely due to the minimal disturbance of the hydraulic and thermal boundary layers when compared to the other designs. The nonuniform flux boundary condition appears to behave more like a uniform flux boundary condition, rather than a uniform temperature boundary condition. For the elliptical tubes, there was more heat transfer when the heat flux was applied normal to the major axis.

3. A non-uniform heat flux boundary condition was applied to each geometry, and normal to both the major and minor axes of the elliptical tubes. It was found that it behaved more like a uniform temperature boundary condition. In the case of elliptical tubes, aligning the flux to be normal with the major axis produced a higher $N u$ than in the other case.

Future work will include producing some or all of these tubes for experimental validation. Except for the inner twisted tape design, it is expected that some form of additive manufacturing will be used in order to assess 


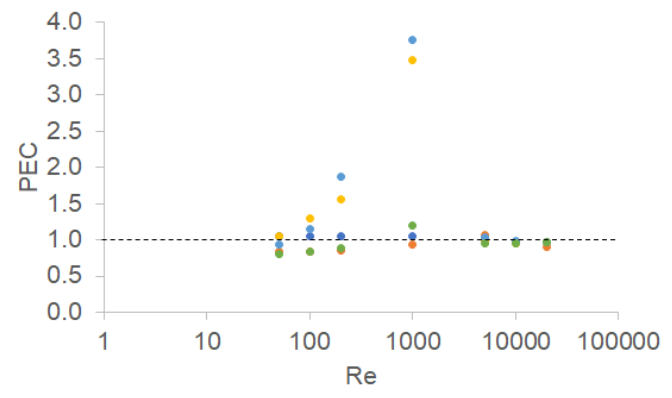

- Ellipse • Airfoil pin fin - TT • TT outer • TT inner

(a) isothermal

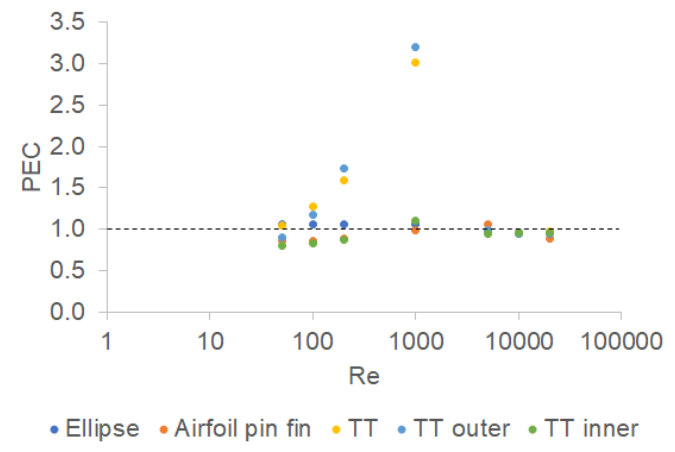

(b) isoflux

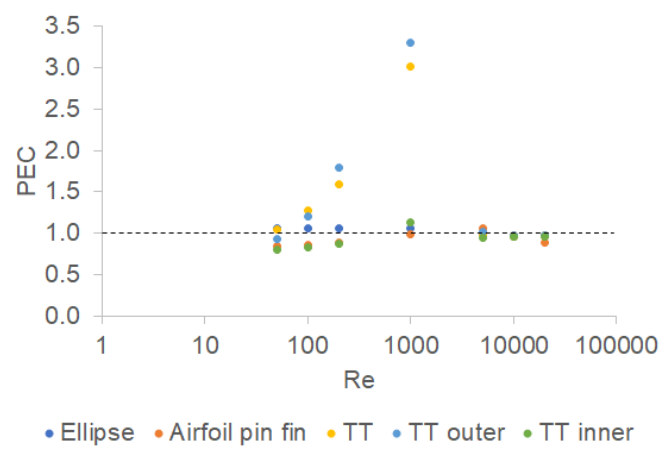

(c) solar

Fig. 7 PEC versus Re for different thermal boundary conditions.

the feasibility of the given manufacturing technique for producing enhanced tubes for heat exchangers. Once these models have been validated, parametric studies can be performed to assess the impact of various design parameters on the PEC of a given design. Of particular interest is the impact of differing airfoil pin geometries and spacings in a given tube to determine their impact on the overall PEC.

\section{ACKNOWLEDGMENTS}

The authors acknowledge the support provided by DOE Solar Program Office and the Technology Manager, Dr. Rajgopal Vijaykumar. The support for the project is provided under DOE solar desalination program (DE-FOA-0001778 - 1686)

\section{REFERENCES}

[1] Abolarin, S. M., Everts, M., and Meyer, J. P., "Heat transfer and pressure drop characteristics of alternating clockwise and counter clockwise twisted tape inserts in the transitional flow regime," International Journal of Heat and Mass Transfer, 133, pp. 203-217, (2019).

[2] Andrade, F., Moita, A. S., Nikulin, A., Moreira, A. L. N., and Santos, H., "Experimental investigation on heat transfer and pressure drop of internal flow in corrugated tubes," International Journal of Heat and Mass Transfer, 140, pp. 940-955, (2019).

[3] Ansys Inc., (2016). Fluent Theory Guide. SAP IP, Inc.

[4] Aroonrat, K., Jumpholkul, C., Leelaprachakul, R., Dalkilic, A. S., Mahian, O., and Wongwises, S., "Heat transfer and singlephase flow in internally grooved tubes," International Communications in Heat and Mass Transfer, 42, pp. 62-68, (2013).

[5] Bhuiya, M. M. K., Chowdhury, M. S. U., Saha, M., and Islam, M. T., "Heat transfer and friction factor characteristics in turbulent flow through a tube fitted with perforated twisted tape inserts," International Communications in Heat and Mass Transfer, 46, pp. 49-57, (2013).

[6] Chang, S. W., Liou, T.-M., and Jiang, Y.-R., "Thermal performances of parallelogram channels with skewed ribs and tilted three dimensional fillets," International Journal of Heat and Mass Transfer, 96, pp. 548-564, (2016). 
[7] Cui, X., Guo, J., Huai, X., Cheng, K., Zhang, H., and Xiang, M., "Numerical study on novel airfoil fins for printed circuit heat exchanger using supercritical co2," International Journal of Heat and Mass Transfer, 121, pp. 354-366, (2018).

[8] Hasanpour, A., Farhadi, M., and K.Sedighi, "A review study on twisted tape inserts on turbulent flow heat exchangers: The overall enhancement ratio criteria," International Communications in Heat and Mass Transfer, 55, pp. 53-62, (2015).

[9] He, Y., Liu, L., Li, P., and Ma, L., "Experimental study on heat transfer enhancement characteristics of tube with cross hollow twisted tape inserts," Applied Thermal Engineering, 131, pp. 743-749, (2018).

[10] Ho, J. Y., Wong, K. K., Leong, K. C., and Wong, T. N., "Convective heat transfer performance of airfoil heat sinks fabricated by selective laser melting," International Journal of Thermal Sciences, 114, pp. 213-228, (2017).

[11] Lim, K. Y., Hung, Y. M., and Tan, B. T., "Performance evaluation of twisted-tape insert induced swirl flow in a laminar thermally developing heat exchanger," Applied Thermal Engineering, 121, pp. 652-661, (2017).

[12] Liu, S. and Sakr, M., "A comprehensive review on passive heat transfer enhancements in pipe exchangers," Renewable and Sustainable Energy Reviews, 19, pp. 64-81, (2013).

[13] Liu, Y., Rao, Y., and Weigand, B., "Heat transfer and pressure loss characteristics in a swirl cooling tube with dimples on the tube inner surface," International Journal of Heat and Mass Transfer, 128, pp. 54-65, (2019).

[14] Nakhchi, M. E. and Esfahani, J. A., "Numerical investigation of rectangular-cut twisted tape insert on performance improvement of heat exchangers," International Journal of Thermal Sciences, 138, pp. 75-83, (2019).

[15] Patankar, S. V., Numerical Heat Transfer and Fluid Flow, CRC Press, (1980).

[16] Piriyarungrod, N., Eiamsa-ard, S., Thianpong, C., Pimsarn, M., and Nanan, K., "Heat transfer enhancement by tapered twisted tape inserts," Chemical Engineering and Processing: Process Intensification, 96, pp. 62-71, (2015).

[17] Ramakumar, B. V. N., Arsha, J. D., and Tayal, P., "Tapered twisted tape inserts for enhanced heat transfer," Journal of Heat Transfer, 138(1), pp. 011901-011901-9, (2015).

[18] Varun, Garg, M. O., Nautiyal, H., Khurana, S., and Shukla, M. K., "Heat transfer augmentation using twisted tapes inserts: A review," Renewable and Sustainable Energy Reviews, 63, pp. 193-225, (2016).

[19] Webb, R. L., "Performance evaluation criteria for use of enhanced heat transfer surfaces in heat exchanger design," International Journal of Heat and Mass Transfer, 24(4), pp. 715-726, (1981).

[20] Yakhot, V., Orszag, S. A., Thangam, S., Gatski, T. B., and Speziale, C. G., "Development of turbulence models for shear flows by a double expansion technique," Physics of Fluids A: Fluid Dynamics, 4(7), pp. 1510-1520, (1992). 